Sebastian Bauer* und Fernando Puente León

\title{
Methoden zur hyperspektralen Bildverarbeitung unter Einbezug von Nachbarschaftsinformation
}

\author{
Methods for hyperspectral image processing incorporating adjacency information
}

https://doi.org/10.1515/teme-2019-0003

Eingang 4. Januar 2019; angenommen 19. Februar 2019

Zusammenfassung: Hyperspektrale Bildgewinnung und -verarbeitung ermöglicht die berührungslose Untersuchung einer Vielzahl von Materialien und Produkten. Die Anwendungsgebiete reichen dabei von der Beurteilung der Lebensmittelsicherheit über die medizinische Bildgebung bis hin zur industriellen Sortierung. Während Hyperspektraluntersuchungen aufgrund der teuren Hardware und komplexen Datenverarbeitung bis vor einigen Jahren hauptsächlich im Labor stattfanden, haben die technischen Entwicklungen der letzten Jahre dazu geführt, dass sie mittlerweile echtzeitfähig sind und inline vorgenommen werden können.

Dieser Artikel beschäftigt sich mit Methoden zur Verarbeitung und Informationsextraktion von Hyperspektralbildern, hauptsächlich der Entrauschung und der spektralen Entmischung. Die vorgestellten Methoden beziehen die inhärent enthaltene, aber meist ignorierte, Nachbarschaftsinformation mit ein.

Schlagwörter: Berührungslose Messung, Hyperspektralbild, Bildgewinnung, Spektrale Entmischung, Entrauschung.

Abstract: Hyperspectral imaging and processing allows for non-contact analysis of a plethora of materials and products. Applications can be found for example in food safety, medical imaging and industrial sorting processes. Due to expensive hardware and complex data processing, hyperspectral analysis was mainly limited to a laboratory environment up until a couple of years ago. In the meantime, technical progress has led to real-time inline applications of hyperspectral analysis.

This article focuses on methods for hyperspectral image processing and information extraction, namely denoising and spectral unmixing, that incorporate adja-

\footnotetext{
*Korrespondenzautor: Sebastian Bauer, University of Wisconsin - Madison, Madison, WI, USA; und Karlsruher Institut für Technologie, Institut für Industrielle Informationstechnik, Karlsruhe, Germany, E-Mail: sebastian.bauer@kit.edu

Fernando Puente León, Karlsruher Institut für Technologie, Institut für Industrielle Informationstechnik, Karlsruhe, Germany
}

cency information. Most existing algorithms ignore this inherent property of hyperspectral images.

Keywords: Non-contact measurement, hyperspectral image, image acquisition, spectral unmixing, denoising.

\section{Einleitung}

Hyperspektrale Bildgewinnung, manchmal auch bildgebende Spektroskopie genannt, liefert für jedes Pixel einer betrachteten Szene ein dicht abgetastetes Spektrum. Es steht somit ein dreidimensionaler Datenwürfel mit zwei räumlichen und einer spektralen Dimension zur Verfügung. Diese Art der Bildgewinnung ist zwar bereits über 100 Jahre alt [1], aber erfuhr erst Mitte der 80er Jahre durch die Verwendung in der Fernerkundung $[2,3,4]$ einen weithin beachteten Aufschwung. Die mittels luft- oder satellitengestützter Kameras aufgenommenen Bilder der Erdoberfläche oder der Erdatmosphäre [5] finden vielfältigen Nutzen beispielsweise zur Mineralerkundung [6], Vegetationsüberwachung [7] und Meeresanalyse [8]. Sie ermöglichen die Lösung von Fragestellungen, die mit herkömmlichen Grauwert- oder Farbbildern nicht lösbar sind. Allerdings waren und sind mit hyperspektraler Bildgebung wesentliche Nachteile wie der große Hardwareaufwand sowie die hohen Kosten und das signifikante Datenvolumen, das zur Informationsextraktion weiter verarbeitet werden muss, verbunden. Die folgende technische Entwicklung von Hyperspektralkameras sowie die damit verbundenen sinkenden Kosten ermöglichten deren Laboreinsatz beispielsweise in der medizinischen Diagnostik $[9,10]$ oder zur Lebensmittelsicherheit [11, 12, 13]. Erste kommerzielle Geräte zur hyperspektralen Inlineuntersuchung sind mittlerweile verfügbar, siehe beispielsweise $[14,15,16]$. Parallel zur Evolution der Hardware entwickeln sich die Methoden zur Informationsextraktion weiter. Oftmals werden chemometrische Methoden auf Hyperspektralbilder angewendet bzw. speziell für diese entwickelt $[17,18]$. Auf weitere Methoden wird im Folgenden noch genauer eingegangen. 
Im Unterschied zu Multispektralbildern, bei denen wenige Spektralbereiche mit unterschiedlichen Abständen zueinander zu jeweils einem Wellenlängenkanal zusammengefasst werden, tasten Hyperspektralkameras das Spektrum gleichmäßig ab und liefern dabei bis zu mehrere hundert Wellenlängenkanäle. Die gleichzeitige Gewinnung von räumlicher und spektraler Information kann dabei auf vielfältige Weise erfolgen. Am weitesten verbreitet sind [19]

- Räumliches Scannen: Die zu untersuchende Fläche wird von einem Punktspektrometer oder einer Zeilenkamera in Verbindung mit einem dispersiven Element zwei- bzw. eindimensional abgetastet.

- Spektrales Scannen: Mittels Hardware- oder programmierbaren Filtern wird aus dem vollständigen Spektrum der interessierende Spektralkanal ausgeschnitten und mittels eines herkömmlichen 2D-Sensors ein Bild angefertigt. Der Prozess wird für alle Spektralkanäle wiederholt.

- Sogenannte Schnappschuss-Aufnahme: Diese relativ neuen Techniken liefern in einem einzigen Aufnahmevorgang einen vollständigen Datenwürfel. Dabei wird das Licht beispielsweise spektral aufgespalten und für jeden Farbkanal ein separates Teilbild auf demselben Sensor abgebildet. Alternativ kommt Compressive Sensing zum Einsatz: Das von der Szene ausgesandte Licht wird gleichzeitig räumlich und spektral moduliert und nach der Aufzeichnung wird mithilfe von Rekonstruktionsmethoden der vollständige Datenwürfel berechnet [20].

Hyperspektraluntersuchungen finden nicht nur im sichtbaren Wellenlängenbereich, sondern auch im ultravioletten und infraroten statt. Die Auswahl des Spektralbereichs hängt dabei oftmals von der gegebenen Aufgabenstellung und den damit verbundenen spektralen Charakteristika der Proben ab. Auch Fluoreszenz kann mittels Hyperspektralanalyse erfasst werden, beispielsweise zur Materialsortierung [21].

Die Methoden zur Informationsextraktion aus Hyperspektralbildern lassen sich im Wesentlichen in die folgenden Gruppen unterteilen:

- Klassifikation: Basierend auf spektralen und ggf. räumlich-spektralen Merkmalen weist ein automatisierter Klassifikator jedes Pixel einer vordefinierten Klasse zu. Während dabei meist davon ausgegangen wird, dass jedes Pixel ausschließlich zu einer Klasse gehört, existieren auch Ansätze zur Klassifikation gemischter Pixel [22].

- Zielentdeckung (engl.: target detection): Aufgrund der großen Entfernungen in der Fernerkundung ist die räumliche Auflösung von Hyperspektralbildern meist sehr gering, sodass jedes einzelne Pixel Kantenlängen im Bereich von einem bis dutzenden Metern besitzt. Dadurch besteht ein Pixelspektrum nicht aus einem einzelnen Reinspektrum, z. B. dem von Gras, sondern setzt sich aus verschiedenen Teilspektren zusammen. Methoden der Zielentdeckung liefern dabei für jedes Pixel die qualitative Aussage, ob ein bestimmtes Zielspektrum im Mischspektrum vorhanden ist [23].

- Spektrale Entmischung: Während die Zielentdeckung nur eine qualitative Aussage liefert, ist die quantitative Aussage das Ziel der spektralen Entmischung (engl.: spectral unmixing). Dabei wird jedes Pixelspektrum in die Reinspektren sowie deren Anteile zerlegt. Sind die Reinspektren bekannt, spricht man von überwachter Entmischung, während unüberwachte Entmischung sowohl die Reinspektren als auch die Anteile liefert.

Die Restauration von Hyperspektralbildern vor der Informationsextraktion umfasst neben beispielsweise der Korrektur defekter Pixel [24] und der passenden Ausrichtung der einzelnen Spektralbänder vor allem die Entrauschung.

Alternativ zur Verarbeitung von Hyperspektralbildern zur Informationsextraktion ist es möglich, optische Spektralfilter so zu programmieren, dass die gesuchte Information, beispielsweise die Materialanteile, unmittelbar als 2D-Grauwertbild verfügbar ist. Erste Ansätze sind z. B. in $[25,26,27]$ beschrieben und nicht Teil der folgenden Betrachtungen.

In diesem Artikel wird zum einen ein neuartiger Entrauschungsalgorithmus vorgestellt, während zum anderen Methoden zur spektralen Entmischung vorgestellt und diskutiert werden, welche räumliche Information mit einbeziehen. Während diese bei der Entrauschung meist verwendet wird, wird sie bei der spektralen Entmischung bisher nur selten berücksichtigt. Der vorliegende Artikel gliedert sich wie folgt: Nach dieser Einleitung folgt in Abschnitt 2 eine kurze Diskussion zum Stand der Technik der hyperspektralen Bildentrauschung. Außerdem werden zwei Varianten eines neuartigen, leistungsfähigen Entrauschungsalgorithmus vorgestellt. Nach der Präsentation der Ergebnisse dieses Algorithmus geht Abschnitt 4 auf den Einbezug von Nachbarschaftsinformation in die spektrale Entmischung von Hyperspektralbildern ein und stellt neuartige Entmischungsmethoden vor. Abschnitt 5 schließt mit einer Zusammenfassung und einem Ausblick auf die Zukunft von Hyperspektraluntersuchungen. 


\section{Entrauschungsalgorithmus}

Während man Hyperspektralbilder jeweils auf der Ebene von eindimensionalen Pixelspektren oder zweidimensionalen Bändern betrachten und dann herkömmliche Methoden zur 1D-Entrauschung bzw. Bildentrauschung anwenden kann, ist es vorteilhaft, alle drei Dimensionen gleichzeitig mit einzubeziehen. Im Folgenden seien einige bestehende Ansätze beispielhaft genannt. Ein Entrauschungsansatz basiert auf der Erweiterung des zweidimensionalen Total Variation-Ansatzes (TV) [28] auf die dritte (spektrale) Dimension durch eine weitere 1DFilterung [29]. Da er räumliche und spektrale Schwankungen von Signal und Rauschen ignoriert, haben Yuan et al. [30] eine adaptive TV-Methode (SSAHTV) entworfen. Qian et al. [31] haben den Non-local Means-Algorithmus von [32] auf 3D-Bilder erweitert. Auch die anisotrope Diffusion [33] wurde für Hyperspektralbilder angepasst [34]. Des Weiteren wurden Entrauschungsalgorithmen vorgestellt, die auf der Niedrigrang-Approximation basieren [35, 36, 37]. Der von Maggioni et al. [38] entwickelte BlockMatching and 4D Filtering-Algorithmus (BM4D) zur Entrauschung von allgemeinen dreidimensionalen Bildern kann auch auf Hyperspektralbilder angewandt werden. SSAHTV und BM4D werden für den neuen Algorithmus als Vergleichsverfahren herangezogen.

Der hier diskutierte neuartige Ansatz basiert auf der gewichteten Addition der 2D-Teilbilder bei jeder Wellenlänge. Er zeichnet sich durch eine dem Stand der Technik vergleichbare Bildqualität bei gleichzeitig deutlich schnellerer Berechnung aus. Für den Fall, dass das Bildrauschen vornehmlich durch eine Poisson-Verteilung beschrieben werden kann, wurde in [39] eine erste Version des Algorithmus vorgestellt. Poisson-Rauschen dominiert bei schwacher Lichtintensität gegenüber dem als normalverteilt angenommenen Sensorrauschen [40]. Außerdem lässt sich das Rauschen in von EMCCD-Sensoren (Electron Multiplying Charge Coupled Device) aufgenommenen Bildern in guter Näherung als Poisson-verteilt beschreiben [41]. Die Poisson-Variante des Entrauschungsalgorithmus wird im Folgenden auf normalverteiltes Rauschen erweitert. Zum einfacheren Verständnis wird zunächst die PoissonVariante kurz beschrieben.

\subsection{Dominantes Poisson-Rauschen}

Das betrachtete Hyperspektralbild wird im Folgenden als $\mathcal{Y} \in \mathbb{R}^{X \times Y \times L}$ bezeichnet. Hierbei stehen $X$ und $Y$ für die Pixelanzahl in beide räumliche Richtungen sowie $L$ für die
Anzahl der Wellenlängen. Die 2D-Teilbilder bei jeder Wellenlänge sind mit $\mathbf{Y}_{l} \in \mathbb{R}^{X \times Y}(1 \leq l \leq L)$ bezeichnet. Da diese Teilbilder als Matrizen behandelt werden, werden alle Rechenoperationen wie Addition, Subtraktion, Multiplikation, Division, Quadrierung sowie die Berechnungen von Erwartungswert und Varianz elementweise ausgeführt. Zunächst wird die Entrauschung für das Teilbild $\mathbf{Y}_{l}$ eingeführt; die Berechnung für alle anderen Teilbilder erfolgt danach analog.

Im ersten Schritt wird $\mathbf{Y}_{l+1}$ gewichtet auf $\mathbf{Y}_{l}$ addiert. Die Elemente beider Matrizen stellen Realisierungen der Poisson-verteilten Zufallsvariablen $\mathrm{Y}_{l}$ und $\mathrm{Y}_{l+1}$ mit den Mittelwerten $\boldsymbol{\Lambda}_{l}$ und $\boldsymbol{\Lambda}_{l+1}$ dar. Die beiden Zufallsvariablen werden als unkorreliert angenommen. Diese Annahme mag oft verletzt sein, da benachbarte Teilbilder relativ ähnlich sein können, führt in der Praxis aber trotzdem zu guten Ergebnissen. Im Fall der Poisson-Verteilung entspricht der Mittelwert der Varianz. Es soll nun ein Schätzer für die Matrix $\Lambda_{l}$ der tatsächlichen Mittelwerte hergeleitet werden. Da die wahren Bilder der Bänder $l$ und $l+1 \mathrm{im}$ Allgemeinen verschieden sind, soll eine gewichtete Addition mit dem Ansatz

$$
\widehat{\boldsymbol{\Lambda}}_{l}=\frac{\mathrm{Y}_{l}+\mathbf{B}_{l+1} \mathbf{C}_{l+1} \mathrm{Y}_{l+1}}{1+\mathbf{B}_{l+1}}
$$

verfolgt werden. Die Matrizen $\mathbf{B}_{l+1}$ und $\mathbf{C}_{l+1}$ stellen ortsabhängige Konstanten dar. An den Schätzer wird die Forderung der Erwartungstreue gestellt,

$$
\mathrm{E}\left\{\widehat{\mathbf{\Lambda}}_{l}\right\}=\frac{\boldsymbol{\Lambda}_{l}+\mathbf{B}_{l+1} \mathbf{C}_{l+1} \boldsymbol{\Lambda}_{l+1}}{1+\mathbf{B}_{l+1}} \stackrel{!}{=} \boldsymbol{\Lambda}_{l},
$$

woraus die Bedingung

$$
\mathbf{C}_{l+1}=\frac{\Lambda_{l}}{\Lambda_{l+1}}
$$

resultiert. Aufgrund der Erwartungstreue entspricht der mittlere quadratische Fehler (Minimum Squared Error, MSE) des Schätzers seiner Varianz [42]. Um den Fehler zu minimieren und damit die Qualität der Schätzung zu verbessern, wird die Varianz minimiert:

$$
\min _{\mathbf{B}_{l+1}} \operatorname{Var}\left\{\widehat{\boldsymbol{\Lambda}}_{l}\right\}=\min _{\mathbf{B}_{l+1}} \frac{\boldsymbol{\Lambda}_{l}+\mathbf{B}_{l+1}^{2}\left(\frac{\boldsymbol{\Lambda}_{l}}{\boldsymbol{\Lambda}_{l+1}}\right)^{2} \boldsymbol{\Lambda}_{l+1}}{\left(1+\mathbf{B}_{l+1}\right)^{2}} .
$$

Die Konstante $\mathbf{B}_{l+1}$ wird damit zu

$$
\mathbf{B}_{l+1}=\frac{\Lambda_{l+1}}{\Lambda_{l}}
$$

ermittelt. Aus den Gleichungen (1), (3) und (5) wird der vollständige Schätzer zu

$$
\widehat{\Lambda}_{l}=\frac{\mathrm{Y}_{l}+\mathrm{Y}_{l+1}}{1+\frac{\Lambda_{l+1}}{\Lambda_{l}}}
$$


bestimmt. Er besitzt den mittleren quadratischen Fehler

$$
\operatorname{MSE}\left\{\widehat{\boldsymbol{\Lambda}}_{l}\right\}=\operatorname{Var}\left\{\widehat{\mathbf{\Lambda}}_{l}\right\}=\frac{\boldsymbol{\Lambda}_{l}+\boldsymbol{\Lambda}_{l+1}}{\left(1+\frac{\boldsymbol{\Lambda}_{l+1}}{\boldsymbol{\Lambda}_{l}}\right)^{2}}=\frac{1}{1+\frac{\boldsymbol{\Lambda}_{l+1}}{\boldsymbol{\Lambda}_{l}}} \boldsymbol{\Lambda}_{l} .
$$

Da $\frac{1}{1+\frac{\Lambda_{l+1}}{\Lambda_{l}}}<1$ gilt, ist die Varianz bzw. der MSE des Schätzers kleiner als die Varianz $\Lambda_{l}$ der Zufallsvariablen $Y_{l}$ des ursprünglichen Bilds. Leider ist das Verhältnis $\frac{\Lambda_{l+1}}{\Lambda_{l}}$ der wahren Mittelwerte nicht bekannt und muss anderweitig geschätzt werden. Hierzu kommt die räumliche Nachbarschaft ins Spiel: Die beiden ursprünglichen Bilder $\mathbf{Y}_{l}$ und $\mathbf{Y}_{l+1}$ werden unter der Annahme lokaler Ergodizität mit zweidimensionalen Gauß-Filtern geglättet. Diese Operation kann mit der Faltung bzw. der Multiplikation im Frequenzbereich effizient durchgeführt werden und liefert einen rauscharmen Schätzwert für das angesprochene Verhältnis. Das entrauschte Bild wird aus dem Schätzer $\widehat{\Lambda}_{l}(6)$ durch

$$
\widehat{\mathbf{Y}}_{l}=\frac{\mathbf{Y}_{l}+\mathbf{Y}_{l+1}}{1+\frac{\Lambda_{l+1}}{\Lambda_{l}}}
$$

berechnet. Um den Rauschanteil noch weiter zu verringern, werden mittels

$$
\widehat{\mathbf{Y}}_{l}=\frac{\mathbf{Y}_{l}+\mathbf{Y}_{l+1}+\cdots+\mathbf{Y}_{l+Z}}{1+\frac{\Lambda_{l+1}}{\Lambda_{l}}+\cdots+\frac{\Lambda_{l+Z}}{\Lambda_{l}}}
$$

noch $Z$ weitere Teilbilder des Hyperspektralbilds einbezogen. $Z$ kann frei gewählt werden und stellt einen Parameter des Verfahrens dar. Die Breite $N$ des quadratischen Filterkerns zur Gauß-Filterung wird zu

$$
N=1+8 \sigma_{\mathrm{GK}}
$$

gewählt, wobei $\sigma_{\mathrm{GK}}$ der Standardabweichung entspricht und ein weiterer Parameter des Entrauschungsverfahrens ist.

Falls der dominierende Rauschanteil normalverteilt ist, entspricht die Varianz der Zufallsvariablen nicht länger dem Mittelwert. Die dadurch notwendige Betrachtung wird im Folgenden durchgeführt.

\subsection{Dominantes Gauß’sches Rauschen}

Auch für normalverteiltes Rauschen gilt ausgehend von (1) und (2) weiterhin

$$
\mathbf{C}_{l+1}=\frac{\Lambda_{l}}{\Lambda_{l+1}}
$$

Die Varianz des Schätzers berechnet sich hingegen zu

$$
\operatorname{Var}\left\{\widehat{\mathbf{\Lambda}}_{l}\right\}=\frac{\operatorname{Var}\left\{\mathrm{Y}_{l}\right\}+\operatorname{Var}\left\{\mathrm{Y}_{l+1}\right\} \mathbf{B}_{l+1}^{2}\left(\frac{\boldsymbol{\Lambda}_{l}}{\boldsymbol{\Lambda}_{l+1}}\right)^{2}}{\left(1+\mathbf{B}_{l+1}\right)^{2}} .
$$

Analog zu den vorigen Betrachtungen für Poisson-Rauschen werden die Herleitungen für normalverteiltes Rauschen durchgeführt, sodass der Schätzer in diesem Fall zu

$$
\widehat{\boldsymbol{\Lambda}}_{l}=\frac{Y_{l}+\frac{\operatorname{Var}\left\{Y_{l}\right\}}{\operatorname{Var}\left\{Y_{l+1}\right\}} \frac{\Lambda_{l+1}}{\Lambda_{l}} Y_{l+1}}{1+\frac{\operatorname{Var}\left\{Y_{l}\right\}}{\operatorname{Var}\left\{Y_{l+1}\right\}}\left(\frac{\Lambda_{l+1}}{\Lambda_{l}}\right)^{2}}
$$

resultiert. Der MSE ergibt sich zu

$$
\operatorname{MSE}\left\{\widehat{\boldsymbol{\Lambda}}_{l}\right\}=\operatorname{Var}\left\{\widehat{\boldsymbol{\Lambda}}_{l}\right\}=\frac{1}{1+\frac{\operatorname{Var}\left\{Y_{l}\right\}}{\operatorname{Var}\left\{Y_{l+1}\right\}}\left(\frac{\boldsymbol{\Lambda}_{l+1}}{\boldsymbol{\Lambda}_{l}}\right)^{2}} \operatorname{Var}\left\{\mathrm{Y}_{l}\right\},
$$

und es ist wiederum ersichtlich, dass die Varianz des Schätzers geringer als die Rauschvarianz im ursprünglichen Bild $Y_{l}$ ist. Das unbekannte Verhältnis der wahren Mittelwerte wird genau wie im Fall von Poisson-Rauschen mittels Gauß-Filterung berechnet. Die Varianzen $\operatorname{Var}\left\{\mathrm{Y}_{l}\right\}$ und $\operatorname{Var}\left\{\mathrm{Y}_{l+1}\right\}$ müssen innerhalb einer gewissen Umgebung $\mathcal{N}(x, y)$ um das jeweils betrachtete Pixel mit der Stichprobenvarianz

$$
\begin{aligned}
\operatorname{Var}\left\{\mathrm{Y}_{l, x y}\right\} & =\frac{1}{N^{2}-1} \sum_{m, n \in \mathcal{N}(x, y)}\left(\mathbf{Y}_{l, m n}-\overline{\mathbf{Y}}_{l, x y}\right)^{2} \\
& =\frac{1}{N^{2}-1} \sum_{m, n \in \mathcal{N}(x, y)} \mathbf{Y}_{l, m n}^{2}-\frac{N^{2}}{N^{2}-1} \cdot \overline{\mathbf{Y}}_{l, x y}^{2}
\end{aligned}
$$

geschätzt werden. Dabei steht $\mathbf{Y}_{l, x y}$ für den Bildwert an der Position $(x, y)$ des Bands $l$. Die Umgebung $\mathcal{N}(x, y)$ besitzt eine quadratische Form der Größe $N \times N$ um $(x, y)$ und entspricht dem Gauß-Kern (10). Für die Mittelwertschätzung in (15) darf nicht die Gauß-Filterung verwendet werden, da hierbei eine andere Gewichtung als von der Formel für die Stichprobenvarianz gefordert verwendet wird. Erst nach vollständiger Berechnung von (16) wird die geschätzte Varianz mittels Gauß-Filterung geglättet.

Nach (13) wird, analog zu (8) im Poisson-Fall, das entrauschte Bild mittels

$$
\widehat{\mathbf{Y}}_{l}=\frac{\mathbf{Y}_{l}+\frac{\operatorname{Var}\left\{Y_{l}\right\}}{\operatorname{Var}\left\{Y_{l+1}\right\}} \frac{\Lambda_{l+1}}{\Lambda_{l}} \mathbf{Y}_{l+1}}{1+\frac{\operatorname{Var}\left\{Y_{l}\right\}}{\operatorname{Var}\left\{Y_{l+1}\right\}}\left(\frac{\Lambda_{l+1}}{\Lambda_{l}}\right)^{2}}
$$

berechnet. Der gleiche Prozess wird nacheinander für alle anderen Bänder $l$ durchgeführt, wobei wie im Poisson-Fall auch jeweils $Z$ weitere Teilbilder verwendet werden. 


\section{Ergebnisse der Entrauschung}

Wie bereits angesprochen, wurden die Poisson-Variante des Entrauschungsalgorithmus und Ergebnisse seiner Anwendung auf hyperspektrale Fluoreszenzbilder in [39] vorgestellt. Weitere Ergebnisse mit Fluoreszenzbildern sind in [43] zu finden.

Die Gauß-Variante des Algorithmus wird beispielhaft an einem Bild aus der Fernerkundung demonstriert. Dieses wurde mit dem HYDICE-Sensor (HYperspectral Digital Imagery Collection Experiment) aufgenommen und bildet die National Mall in Washington, DC ab [44]. Das gesamte Bild umfasst $1280 \times 307$ Pixel und 191 Bänder. Hier wird nur ein Ausschnitt der Größe $251 \times 201$ betrachtet, der ungefähr den Bereich umfasst, der auch in [30] untersucht wurde. Alle Wellenlängen wurden in die Entrauschung mit einbezogen. Das ursprüngliche Bild wird mit künstlichem Gauß'schen Rauschen versehen, wobei das SNR 10, 20 und $30 \mathrm{~dB}$ betrug. Zusätzlich wurde in einer vierten Variante das SNR der einzelnen Bänder zufällig zwischen 15 und $25 \mathrm{~dB}$ variiert. Der hier neu vorgestellte Algorithmus wird mit SSAHTV [30] und dem BlockMatching and 4D Filtering-Algorithmus (BM4D) [38] verglichen. BM4D ist eine Erweiterung des leistungsstarken Block-Matching and 3D Filtering-Algorithmus [45] auf dreidimensionale Bilder. Der SSAHTV-Quellcode wurde uns freundlicherweise von den Autoren der Veröffentlichung zur Verfügung gestellt, der BM4D-Quellcode ist online verfügbar [46]. Weitere Details sowie eine genauere Untersuchung des Entrauschungsergebnisses sind in [43] enthalten. Tabelle 1 gibt die Entrauschungsqualität als MPSNR (Mean Peak Signal to Noise Ratio), also mittleres bandweises SNR, an. Außerdem wird die Berechnungszeit aufgeführt. Es ist zu erkennen, dass der neue Algorithmus mit die beste Entrauschungsqualität liefert, dabei aber 25 -fach schneller als der qualitativ ähnliche BM4D-Algorithmus abläuft. Dies liegt daran, dass der neue Algorithmus im
Wesentlichen nur auf der effizient zu berechnenden Glättung mit Gauß-Filtern, also der Faltung, und der Addition von Teilbildern beruht. BM4D hingegen basiert auf einem deutlich komplexeren Verfahren; so sucht der BM4D im Bild zunächst nach ähnlichen Bildbereichen, um diese gemeinsam weiterzuverarbeiten. Für weitere Details siehe [38]. Abbildung 1 veranschaulicht die Entrauschungsergebnisse am Beispiel eines Bandes.

\section{Spektrale Entmischung}

Wie bereits in der Einleitung beschrieben, hat die spektrale Entmischung das Ziel, die gemischten Pixel eines aufgezeichneten Hyperspektralbilds in die Reinstoffe sowie ihre jeweiligen Anteile zu zerlegen. Oftmals wird dabei vom sogenannten linearen Mischmodell ausgegangen:

$$
\mathbf{Y}=\mathbf{M A}+\mathbf{\Psi}
$$

Hierbei enthält die Matrix $\mathbf{Y} \in \mathbb{R}^{L \times I}$ spaltenweise die Pixelspektren des Hyperspektralbilds; das dreidimensionale Bild ist also in eine zweidimensionale Darstellung überführt worden. $L$ steht wie bei der Entrauschung für die Anzahl der Wellenlängen und $I$ beschreibt die Gesamtanzahl der Pixel, $I=X \cdot Y$. Die Matrix $\Psi$ enthält den Modellierungsfehler und Rauschen. Die Matrix $\mathbf{Y}$ setzt sich zusammen aus der Reinspektrenmatrix $\mathbf{M} \in \mathbb{R}^{L \times R}$ und der Anteilsmatrix $\mathbf{A} \in \mathbb{R}^{R \times I}$, wobei $R$ die Anzahl der Reinstoffe angibt. Die spektrale Entmischung muss also das gemessene Hyperspektralbild $\mathbf{Y}$ in diese beiden Matrizen zerlegen. Die Berechnung dieser Zerlegung erfolgt oftmals mit Methoden der Nichtnegativen Matrixzerlegung (Nonnegative Matrix Factorization, NMF) [47], nämlich durch Minimierung des Gütemaßes

$$
Q_{\text {Bild }}(\mathbf{M}, \mathbf{A})=\|\mathbf{M A}-\mathbf{Y}\|_{\mathrm{F}}^{2} .
$$

Tab. 1: MPSNR (Mean Peak Signal to Noise Ratio)-Werte aller Algorithmen angewandt auf das National Mall-Bild (in dB). Bei allen Rauschniveaus liefert der neue Algorithmus ähnliche Werte wie BM4D, meist bessere. Allerdings ist die Berechnung ungefähr 25-fach schneller als bei BM4D. Der Parameter $\sigma$ von BM4D wird zu 0 gewählt, was bedeutet, dass der Algorithmus das Rauschniveau selbst schätzt. Die Spalte $\gamma$ gibt den optimalen Parameterwert für SSAHTV an. Die verwendete Software ist MATLAB, die verwendete Hardware ein 3.5 GHz i7-Prozessor

\begin{tabular}{|c|c|c|c|c|c|c|c|c|c|c|}
\hline \multirow{2}{*}{ SNR } & \multirow{2}{*}{$\begin{array}{r}\text { Verr. Bild } \\
\text { MPSNR }\end{array}$} & \multicolumn{4}{|c|}{ Neuer Algorithmus } & \multicolumn{2}{|l|}{ BM4D } & \multicolumn{3}{|l|}{ SSAHTV } \\
\hline & & MPSNR & $\mathrm{Z} / 2$ & $\sigma_{\mathrm{GK}}$ & $t / \mathrm{s}$ & MPSNR & $t / \mathrm{s}$ & MPSNR & $\gamma$ & $t / \mathrm{s}$ \\
\hline $10 \mathrm{~dB}$ & 21,58 & 33,22 & 14 & 18 & 25,7 & 32,31 & 494,9 & 27,45 & 2 & 93,6 \\
\hline $20 \mathrm{~dB}$ & 31,58 & 39,60 & 6 & 20 & 18,7 & 39,50 & 498,8 & 34,48 & 10 & 21,6 \\
\hline $30 \mathrm{~dB}$ & 41,58 & 45,69 & 2 & 21 & 15,0 & 46,77 & 500,4 & 42,50 & 50 & 11,2 \\
\hline var. & 32,07 & 39,46 & 6 & 21 & 19,7 & 39,35 & 498,0 & 34,62 & 10 & 20,8 \\
\hline
\end{tabular}
mit 16 GB RAM. 

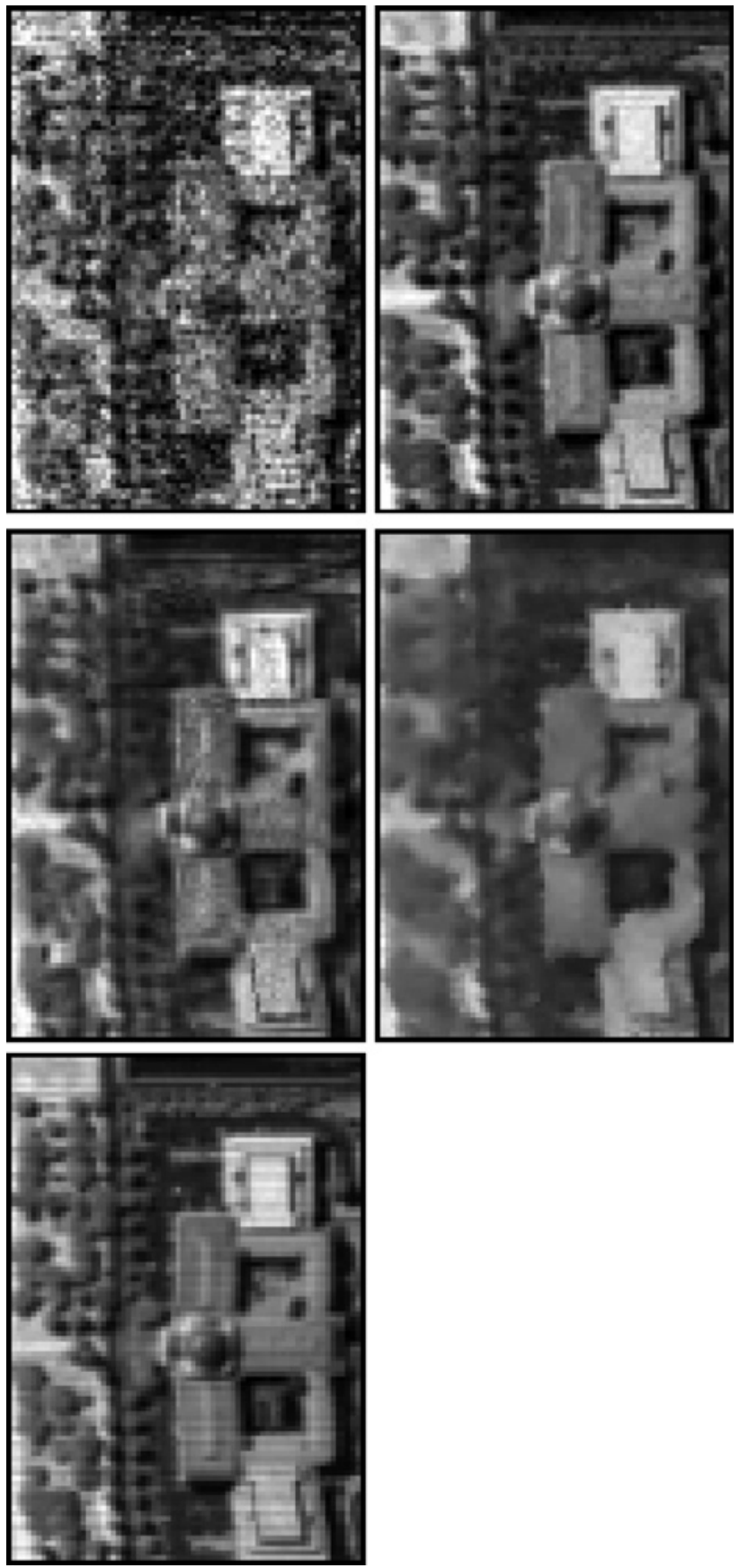

Abb. 1: Detailansicht eines Ausschnitts von Band 102 des National Mall-Bildes bei $10 \mathrm{~dB}$ SNR. Links oben: verrauschtes Bild, rechts: neuer Algorithmus. Mittlere Reihe links: BM4D, rechts: SSAHTV. Unten: tatsächliches Bild. Man beachte die Detailtreue des neuen Algorithmus, während die beiden anderen Algorithmen große Bildbereiche verschmieren.

$\|\cdot\|_{F}$ steht hierbei für die Frobeniusnorm. Die Optimierung geschieht durch alternierende Minimierung nach $\mathbf{M}$ für konstantes A und umgekehrt. Da sowohl die Reinspektren als auch die Materialanteile nicht negativ sein dürfen, wird diese Nebenbedingung mit in die Optimierung einbezogen. Außerdem müssen sich alle Materialanteile eines Pixels jeweils zu 1 aufsummieren. Das Gütemaß (19) ist bikonvex, d.h. es ist konvex jeweils in $\mathbf{M}$ und $\mathbf{A}$, aber nicht gleichzeitig konvex für beide Matrizen. Aus diesem Grund wird es oftmals um weitere Terme erweitert, die Zusatzwissen in Form von Bedingungen an Reinstoffe $\left(\mathcal{R}_{\mathrm{S}}(\mathbf{M})\right)$ bzw. Materialanteile $\left(\mathcal{R}_{\mathrm{r}}(\mathbf{A})\right)$ einbeziehen:

$$
Q(\mathbf{M}, \mathbf{A})=Q_{\text {Bild }}(\mathbf{M}, \mathbf{A})+\mu_{\mathrm{s}} \mathcal{R}_{\mathrm{s}}(\mathbf{M})+\mu_{\mathrm{r}} \mathcal{R}_{\mathrm{r}}(\mathbf{A}) .
$$

Diese werden mit den Regularisierungsparametern $\mu_{\mathrm{s}}$ und $\mu_{\mathrm{r}}$ gewichtet. Das lineare Mischmodell vernachlässigt Interaktionen zwischen den einzelnen Reinspektren; diese können mit nichtlinearen Mischmodellen [48] modelliert werden, die allerdings nicht Gegenstand dieser Betrachtungen sind.

\subsection{Einbezug räumlicher Nachbarschaft}

Das Überblickspaper [49] aus dem Jahr 2012 enthält eine Reihe von Beispielen für spektrale und räumliche Regularisierungsterme $\mathcal{R}_{\mathrm{s}}(\mathbf{M})$ bzw. $\mathcal{R}_{\mathrm{r}}(\mathbf{A})$ in Gl. (20). Bis zu diesem Zeitpunkt wurde meist mit spektralen Regularisierungstermen gearbeitet, die zweifellos einen sehr großen Einfluss auf die Entmischungsqualität haben. Allerdings wird in der Referenz auch betont, dass die Verwendung von zusätzlicher Kontextinformation sehr vorteilhaft für die Analyse hyperspektraler Daten sein kann. Es werden einige Beispiele von Entmischungsalgorithmen genannt, die räumliche Information mit einbeziehen. Nach 2012 sind noch einige weitere solche Algorithmen hinzugekommen.

Während das übergeordnete Ziel des Einbezugs räumlicher Information natürlich die Verbesserung der Entmischungsqualität ist, kann diese auf unterschiedliche Art und Weise erreicht werden. Methoden wie z. B. die in [50] beschriebene haben das Ziel, den negativen Einfluss von Spektrenvariabilität zu verringern. Dabei wird berücksichtigt, dass aufgrund von unterschiedlichen Einflüssen wie zum Beispiel Objektgeometrie oder Lichtbedingungen [51] das Spektrum eines Reinstoffs nicht über das gesamte Bild als konstant angenommen werden kann. Ein anderer Ansatz wird beispielsweise in [52] verfolgt; hierbei wird die Nebenbedingung gestellt, dass die Anteile benachbarter Pixel möglichst ähnlich sein sollen. Dieser Ansatz wird im Folgenden noch genauer diskutiert. Einen Überblick zu Methoden zum Einbezug räumlicher Information in die spektrale Entmischung liefert beispielsweise [53]. 
Ausgangspunkt von [52] ist die Erweiterung des zum linearen Mischmodell gehörenden Gütemaßes (19) in Übereinstimmung mit dem allgemeinen Erweiterungsansatz (20), wobei hier nur die Regularisierung eines einzelnen Pixels statt der gesamten Anteilsmatrix A betrachtet wird. An die Materialanteile jedes Pixels $i$ wird die Nebenbedingung gestellt, dass die Anteile der Pixel in einer bestimmten Umgebung $\mathcal{N}(i)$ möglichst ähnlich sein sollen. Dies wird mittels der Summennorm formuliert:

$$
\sum_{j \in \mathcal{N}(i)}\left\|\mathbf{a}_{i}-\mathbf{a}_{j}\right\|_{1} .
$$

Durch diese Nebenbedingung werden konstante Anteilsverläufe gefordert. Somit wird also, ähnlich wie bei TV, eine Minimierung der ersten räumlichen Ableitung bewirkt. Aufgrund der Tatsache, dass dadurch lineare Signalverläufe durch Sprünge approximiert werden, wurde diese Methode von Bauer et al. auf die Minimierung der zweiten räumlichen Ableitung [54] sowie auf eine lineare bzw. quadratische Gewichtung der Abweichung [55] erweitert.

Ein anderer Ansatz zur Verwendung von räumlicher Information wird in Bauer et al. [56] verfolgt. Dabei werden die Pixel des vorliegenden Hyperspektralbilds zunächst aufgrund von räumlichen und ggf. räumlich-spektralen Ähnlichkeitsmaßen geclustert. Im nächsten Schritt wird der Mittelwert der Pixelspektren eines Clusters gebildet, wodurch sich die Anzahl der Spalten der Bildmatrix Y zum Teil deutlich verringert. Dadurch wird die Entmischung deutlich schneller berechnet, während durch die Mittelung der Einfluss des Rauschens deutlich verringert wird. Eine ausführliche Betrachtung des Einflusses von Clustermethode und Clusteranzahl auf Entmischungsqualität und Berechnungszeit ist in [43] zu finden. Diese Methode beeinflusst also gleichzeitig die Entmischungsqualität und die dazu benötigte Berechnungszeit. Im Unterschied dazu wird in Bauer etal. [57] das Konzept der Bildpyramiden zur beschleunigten Berechnung der Entmischung verwendet. Die resultierende Methode erlaubt es, Berechnungszeit und Entmischungsgüte auszutarieren; eine schnelle Berechnung liefert ausbaufähige Ergebnisse, wohingegen eine gute Entmischung mehr Berechnungszeit erfordert.

\subsection{Einbezug globaler 3D-Nachbarschaft}

Bis hierhin wurde nur die räumliche Pixelnachbarschaft diskutiert, in manchen Fällen ergänzt um eine gewisse spektrale Komponente. Dies wirft die Frage auf, wie die Entmischungsqualität von der Verwendung der globalen im Bild vorhandenen 3D-Nachbarschaft profitieren kann, also wie 3D-Nachbarschaftsbeziehungen auf unterschiedlichen Entfernungsskalen genutzt werden können. In der Literatur existieren Ansätze wie z. B. [58], die das Hyperspektralbild nicht in die zweidimensionale Matrix $\mathbf{Y}$ entfalten, sondern es als dreidimensionalen Tensor weiterverarbeiten. Obwohl die Weiterverwendung als Tensor natürlich jedes Bildelement während der nächsten Schritte in seiner ursprünglichen Nachbarschaft belässt, stellt sich die Frage, inwieweit der Tensor-Ansatz die Verwendung von Nachbarschaftsrelationen auf größeren Skalen zulässt. Diese Frage soll hier nicht diskutiert werden; stattdessen soll das in [43] ausführlich vorgestellte Verfahren, welches die gesamte dreidimensionale Nachbarschaft verwendet, kurz umrissen werden.

Ausgangspunkt des Verfahrens ist die Tatsache, dass bestimmte dreidimensionale Transformationen wie beispielsweise die 3D-Kosinustransformation (Discrete Cosine Transform, DCT) genau die gefragte globale dreidimensionale Nachbarschaft der Bildelemente ausnutzen bzw. codieren. Die Anwendung der 3D-DCT liefert einen Würfel von der gleichen Größe wie das ursprüngliche Hyperspektralbild. Der neue Datenwürfel enthält die Koeffizienten $y\left(f_{x}, f_{y}, f_{l}\right)$ der dreidimensionalen Ortsfrequenzen. Diese werden aus den Bildelementen $y(x, y, l)$ berechnet mittels

$$
\begin{aligned}
y\left(f_{x}, f_{y}, f_{l}\right)= & \frac{1}{\sqrt{X Y L}} \sum_{x=0}^{X-1} \sum_{y=0}^{Y-1} \sum_{l=0}^{L-1} y(x, y, l) \\
& \cdot \cos \left(\frac{\pi}{X}\left(x+\frac{1}{2}\right) f_{x}\right) \cos \left(\frac{\pi}{Y}\left(y+\frac{1}{2}\right) f_{y}\right) \\
& \cdot \cos \left(\frac{\pi}{L}\left(l+\frac{1}{2}\right) f_{l}\right) .
\end{aligned}
$$

Aus der Formel ist ersichtlich, dass die Vertauschung von lediglich zwei Bildelementen, z. B. zwei direkt benachbarten, die Summation entlang der drei Würfelrichtungen ändert, da die beiden vertauschten Elemente mit anderen Kosinus-Termen multipliziert werden. Dies gilt für sämtliche Koeffizienten, die dadurch andere Werte erhalten, und auch über große Distanzen zwischen den Elementen hinweg: vertauscht man im Extremfall die Elemente an zwei gegenüberliegenden Ecken des Würfels, so ändern sich auch in diesem Fall die Werte des kompletten DCT-Würfels, siehe auch Abb. 2. Somit enthält dieser die vollständige globale dreidimensionale Nachbarschaftsinformation. Nach dem besten Wissen der Autoren ist so ein Entmischungsverfahren, welches die gesamte Nachbarschaftsinformation verwendet, in der Literatur noch nicht beschrieben worden. Da die Kosinustransformation eine orthogonale Transformation ist, ändert sich der Wert des Gütemaßes (19) durch ihre Anwendung nicht. 

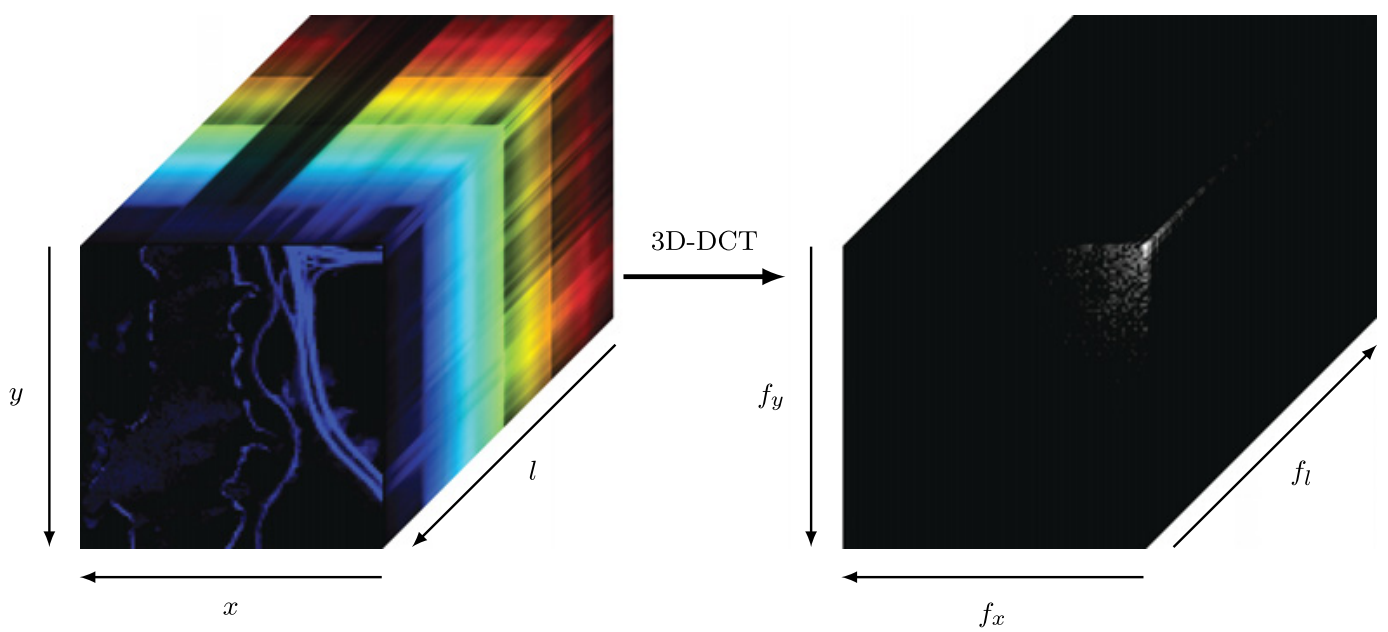

Abb. 2: Datenwürfel eines Bilds aus der Fernerkundung (links) sowie der 3D-DCT-transformierte Würfel mit logarithmischer Grauwertskalierung. Die meiste Signalenergie ist um den Punkt $f_{x}=f_{y}=f_{l}=0$ konzentriert. Durch Vertauschen zweier Bildelemente des ursprünglichen Bilds ändern sich sämtliche Werte des transformierten Würfels.

Aus diesem Grund stützt sich die Berechnung des DCTEntmischungsverfahrens nicht auf sämtliche Frequenzkoeffizienten, sondern nur einen gewissen Anteil der betragsgrößten. Dadurch wird die Spärlichkeit des Hyperspektralbilds ausgenutzt und außerdem ergibt sich ein von (19) verschiedenes Gütemaß. Dieses wird außerdem noch dahingehend verändert, dass die ursprüngliche Reinspektrenmatrix $\mathbf{M}$ in quadratische Blöcke aufgespalten wird, genauso wie auch die transformierte Bildmatrix in Blöcke aufgeteilt wird. Es werden nun mittels des modifizierten Gütemaßes der Form

$$
Q_{\bmod }=\sum_{j}\left\|\mathbf{N}_{j} \mathbf{Y}-\mathbf{A}\right\|_{\mathrm{F}}^{2}
$$

invertierte Teilmatrizen $\mathbf{N}_{j}$ berechnet. Nach erfolgter Optimierung werden diese wiederum blockweise invertiert und zu einer Matrix der alten Größe von M zusammengesetzt. Schlussendlich wird die Gesamtmatrix der inversen 1D-DCT in spektraler Richtung unterzogen und somit eine Schätzung der Reinspektren erhalten. Im Gegensatz zum Ausgangsgütemaß ist das neue Gütemaß (23) konvex, muss aber um Regularisierungsterme zur Vermeidung der Triviallösung $\mathbf{N}=\mathbf{0}$ ergänzt werden. Für weitere Details des Verfahrens sowie für Entmischungsergebnisse sei auf [43] verwiesen.

\section{Zusammenfassung und Ausblick}

In diesem Beitrag wurde der Stand der Technik der hyperspektralen Bildgewinnung und -verarbeitung grob umrissen sowie Verfahren zur Entrauschung und Entmischung unter Einbezug globaler dreidimensionaler Nachbarschaftsinformation vorgestellt. Der neue Entrauschungsalgorithmus erreicht dabei eine ähnliche Ergebnisqualität wie Verfahren aus dem Stand der Technik, allerdings bis zu 25-mal schneller. Generell ist es zu erwarten, dass sich Hyperspektraluntersuchungen aufgrund ihrer Vielseitigkeit und Leistungsfähigkeit in immer mehr Anwendungsgebieten der optischen Inspektion durchsetzen werden. Auch eine Reihe von neuartigen Anwendungsgebieten ist denkbar bzw. sogar schon in der Entwicklung; mehrere Firmen arbeiten bereits an Sensorik und Auswertealgorithmen für mobile Anwendungen. So ließe sich dann mit dem Smartphone der Frischezustand von Lebensmitteln vor dem Kauf in Echtzeit überprüfen.

\section{Literatur}

1. AVIRIS Imaging Spectroscopy. [Online]. Available: https: //aviris.jpl.nasa.gov/html/aviris.spectroscopy.html.

2. A. F. Goetz, G. Vane, J. E. Solomon und B. N. Rock, ,Imaging spectrometry for earth remote sensing ", Science, Vol. 228, Nr. 4704, S. 1147-1153, 1985.

3. A. F. Goetz, , Three decades of hyperspectral remote sensing of the earth: A personal view", Remote Sensing of Environment, Vol.113, S. S5-S16, 2009.

4. M. T. Eismann, Hyperspectral remote sensing, Bellingham: SPIE, 2012.

5. E. Ben-Dor, D. Schläpfer, A. J. Plaza und T. Malthus, „Hyperspectral remote sensing“, Airborne Measurements for Environmental Research: Methods and Instruments, S. 413-456, 2013. 
6. F. F. Sabins, ,, Remote sensing for mineral exploration“, Ore Geology Reviews, Vol. 14, Nr. 3-4, S. 157-183, 1999.

7. P. S. Thenkabail und J. G. Lyon, Hyperspectral remote sensing of vegetation, CRC Press, 2016.

8. K. L. Carder, P. Reinersman, R. F. Chen, F. Muller-Karger, C. O. Davis und M. Hamilton, ,AVIRIS calibration and application in coastal oceanic environments", Remote Sensing of Environment, Vol. 44, Nr. 2-3, S. 205-216, 1993.

9. G. Lu und B. Fei, ,,Medical hyperspectral imaging: a review", Journal of Biomedical Optics, Vol.19, Nr.1, S. 010901-1-010901-23, 2014.

10. H. Grahn und P. Geladi, Techniques and applications of hyperspectral image analysis, John Wiley \& Sons, 2007.

11. D.-W. Sun, Hyperspectral imaging for food quality analysis and control. Elsevier, 2010.

12. G. ElMasry, M. Kamruzzaman, D.-W. Sun und P. Allen, „Principles and applications of hyperspectral imaging in quality evaluation of agro-food products: a review", Critical Reviews in Food Science and Nutrition, Vol. 52, Nr. 11, S. 999-1023, 2012.

13. M. Kopf, R. Gruna, T. Längle und J. Beyerer, „Evaluation and comparison of NIR multi-product calibration methods for Brix prediction", tm - Technisches Messen, Vol. 85, Nr. 3, S. 184-190, 2018.

14. Hyperspektraler Freifall-Sortierer. [Online]. Available: https: //waste-management-world.com/a/best-launches-free-fallhyperspectral-sorting-machine.

15. Industrielle Hochgeschwindigkeits-Hyperspektralkamera. [Online]. Available: https://www.perception-park.com/ hyperspectral-imaging-1000-frames-second.

16. Resonon Hyperspektral-Sortierer. [Online]. Available: https: //www.resonon.com/Products/machine_vision.html.

17. J. M. Amigo, H. Babamoradi und S. Elcoroaristizabal, „Hyperspectral image analysis. A tutorial“, Analytica Chimica Acta, Vol. 896, Supplement C, S. 34-51, 2015.

18. Y. Roggo, A. Edmond, P. Chalus und M. Ulmschneider, „Infrared hyperspectral imaging for qualitative analysis of pharmaceutical solid forms ", Analytica Chimica Acta, Vol. 535, Nr.1, S. 79-87, 2005.

19. A. Gowen, C. O'Donnell, P. Cullen, G. Downey und J. Frias, „Hyperspectral imaging-an emerging process analytical tool for food quality and safety control", Trends in Food Science \& Technology, Vol. 18, Nr. 12, S. 590-598, 2007.

20. R. M. Willett, M. F. Duarte, M. A. Davenport und R. G. Baraniuk, "Sparsity and structure in hyperspectral imaging: Sensing, reconstruction, and target detection ", IEEE Signal Processing Magazine, Vol. 31, Nr. 1, S. 116-126, 2014.

21. S. Bauer und F. Puente León, , Gewinnung und Verarbeitung hyperspektraler Fluoreszenzbilder zur optischen Mineralklassifikation", tm - Technisches Messen, Vol. 82, Nr.1, S. 24-33, 2015.

22. C. Chang, Hyperspectral Imaging: Techniques for Spectral Detection and Classification, Springer US, 2013.

23. C. Chang, Hyperspectral Data Processing: Algorithm Design and Analysis, Wiley, 2013.

24. Y. Xie, Y. Qu, D. Tao, W. Wu, Q. Yuan, W. Zhang et al., „Hyperspectral image restoration via iteratively regularized weighted Schatten p-norm minimization", IEEE Trans. Geoscience and Remote Sensing, Vol. 54, Nr. 8, S. 4642-4659, 2016.
25. W. Krippner, S. Bauer und F. Puente León, „Ortsaufgelöste optische Bestimmung von Materialanteilen in Mischungen", tm - Technisches Messen, Vol. 84, Nr. 3, S. 207-215, 2017.

26. W. Krippner und F. Puente León, ,,Bandbereichswahl und Materialanteilsschätzung mithilfe von Spektralfiltern", tm Technisches Messen, Vol. 85, Nr. 6, S. 454-467, 2018.

27. W. Krippner, S. Bauer und F. Puente León, , Considering spectral variability for optical material abundance estimation", tm-Technisches Messen, Vol. 85, Nr. 3, S. 149-158, 2018.

28. L. I. Rudin, S. Osher und E. Fatemi, ,,Nonlinear total variation based noise removal algorithms", Physica D: Nonlinear Phenomena, Vol. 60, Nr. 1-4, S. 259-268, 1992.

29. H. Zhang, ,Hyperspectral image denoising with cubic total variation model", ISPRS Annals of Photogrammetry, Remote Sensing and Spatial Information Sciences, Vol. 7, S. 95-98, 2012.

30. Q. Yuan, L. Zhang und H. Shen, ,Hyperspectral image denoising employing a spectral-spatial adaptive total variation model", IEEE Transactions on Geoscience and Remote Sensing, Vol. 50, Nr. 10, S. 3660-3677, 2012.

31. Y. Qian, Y. Shen, M. Ye und Q. Wang, ,,3-D nonlocal means filter with noise estimation for hyperspectral imagery denoising ", in IEEE International Geoscience and Remote Sensing Symposium (IGARSS), 2012, S. 1345-1348.

32. A. Buades, B. Coll und J.-M. Morel, „A review of image denoising algorithms, with a new one", Multiscale Modeling \& Simulation, Vol. 4, Nr. 2, S. 490-530, 2005.

33. P. Perona und J. Malik, ,Scale-space and edge detection using anisotropic diffusion", IEEE Transactions on Pattern Analysis and Machine Intelligence, Vol.12, Nr. 7, S. 629-639, 1990.

34. Y. Wang, R. Niu und X. Yu, ,,Anisotropic diffusion for hyperspectral imagery enhancement", IEEE Sensors Journal, Vol. 10, Nr. 3, S. 469-477, 2010.

35. W. He, H. Zhang, L. Zhang und H. Shen, ,Hyperspectral image denoising via noise-adjusted iterative low-rank matrix approximation", IEEE Journal of Selected Topics in Applied Earth Observations and Remote Sensing, Vol. 8, Nr. 6, S. 3050-3061, 2015.

36. H. Zhang, W. He, L. Zhang, H. Shen und Q. Yuan, ,Hyperspectral image restoration using low-rank matrix recovery", IEEE Transactions on Geoscience and Remote Sensing, Vol. 52, Nr. 8, S. 4729-4743, 2014.

37. Y.-Q. Zhao und J. Yang, ,Hyperspectral image denoising via sparse representation and low-rank constraint", IEEE Transactions on Geoscience and Remote Sensing, Vol.53, Nr.1, S. 296-308, 2015.

38. M. Maggioni, V. Katkovnik, K. Egiazarian und A. Foi, ,Nonlocal transform-domain filter for volumetric data denoising and reconstruction", IEEE Transactions on Image Processing, Vol. 22, Nr. 1, S.119-133, 2013.

39. S. Bauer, P. Mackens und F. Puente León, „Entrauschungsalgorithmus für Hyperspektralbilder mit Poisson-Statistik", in XXIX. Messtechnisches Symposium 2015, T. Fröhlich and E. Manske, Hrsg. Berlin: De Gruyter Oldenbourg, 2015, S. 91-98.

40. European Machine Vision Association, EMVA 1288 standard, Release 3.1. [Online]. Available: http://www.emva.org/wpcontent/uploads/EMVA1288-3.1a.pdf.

41. M. Hirsch, R. J. Wareham, M. L. Martin-Fernandez, M. P. Hobson und D. J. Rolfe, „A stochastic model for electron multiplication 
charge-coupled devices-from theory to practice", PloS one, Vol. 8, Nr. 1, 2013.

42. T. Rudas, Handbook of Probability: Theory and Applications, SAGE Publications, 2008.

43. S. Bauer, ,Hyperspectral image unmixing incorporating adjacency information", Dissertation, Karlsruhe, 2018.

44. MultiSpec-Hyperspectral images web page. [Online]. Available: https://engineering.purdue.edu/ biehl/MultiSpec/ hyperspectral.html.

45. K. Dabov, A. Foi, V. Katkovnik und K. Egiazarian, ,,Image denoising by sparse 3-D transform-domain collaborative filtering “, IEEE Transactions on Image Processing, Vol. 16, Nr. 8, S. 2080-2095, 2007.

46. Image and video denoising by sparse 3D transform-domain collaborative filtering web page. [Online]. Available: http: //www.cs.tut.fi/ foi/GCF-BM3D/.

47. A. Cichocki, R. Zdunek, A. Phan und S. Amari, Nonnegative Matrix and Tensor Factorizations: Applications to Exploratory Multi-way Data Analysis and Blind Source Separation, Wiley, 2009.

48. R. Heylen, M. Parente und P. Gader, „A review of nonlinear hyperspectral unmixing methods", IEEE Journal of Selected Topics in Applied Earth Observations and Remote Sensing, Vol. 7, Nr. 6, S. 1844-1868, 2014.

49. J. M. Bioucas-Dias, A. Plaza, N. Dobigeon, M. Parente, Q. Du, P. Gader und J. Chanussot, ,Hyperspectral unmixing overview: Geometrical, statistical, and sparse regression-based approaches", IEEE Journal of Selected Topics in Applied Earth Observations and Remote Sensing, Vol. 5, Nr. 2, S. 354-379, 2012.

50. L. Drumetz, M.-A. Veganzones, S. Henrot, R. Phlypo, J. Chanussot und $C$. Jutten, ,Blind hyperspectral unmixing using an extended linear mixing model to address spectral variability", IEEE Transactions on Image Processing, Vol. 25, Nr. 8, S. 3890-3905, 2016.

51. A. Zare und K. Ho, ,Endmember variability in hyperspectral analysis: Addressing spectral variability during spectral unmixing “, IEEE Signal Processing Magazine, Vol. 31, Nr. 1, S. 95-104, 2014.

52. A. Zymnis, S.-J. Kim, J. Skaf, M. Parente und S. Boyd, „Hyperspectral image unmixing via alternating projected subgradients", in Conference Record of the Forty-First Asilomar Conference on Signals, Systems and Computers, ACSSC, 2007, S. 1164-1168.

53. C. Shi und L. Wang, ,Incorporating spatial information in spectral unmixing: A review", Remote Sensing of Environment, Vol. 149, S. 70-87, 2014.

54. S. Bauer, J. Stefan, M. Michelsburg, T. Längle und F. Puente León, ,, Robustness improvement of hyperspectral image unmixing by spatial second-order regularization", IEEE Transactions on Image Processing, Vol. 23, Nr.12, S. 5209-5221, 2014.

55. S. Bauer, F. Neumann und F. Puente León, ,Spatial regularization for the unmixing of hyperspectral images", in Automated Visual Inspection and Machine Vision, Ser. Proceedings of SPIE, Vol. 9530, 2015.
56. S. Bauer, E. Winterbauer und F. Puente León, „Accelerating spectral unmixing by using clustered images", in IEEE International Conference on Image Processing (ICIP), Beijing, China, 2017.

57. S. Bauer und F. Puente León, ,Using image pyramids for the acceleration of spectral unmixing based on nonnegative matrix factorization", in: 8th IEEE Workshop on Hyperspectral Image and Signal Processing: Evolution in Remote Sensing (WHISPERS), Los Angeles, CA, S. 733-737, 2016.

58. Q. Zhang, H. Wang, R. Plemmons und V. Paul Pauca, ,Spectral unmixing using nonnegative tensor factorization", in Proceedings of the 45th annual southeast regional conference. ACM, 2007, S. 531-532.

\section{Autoreninformationen}

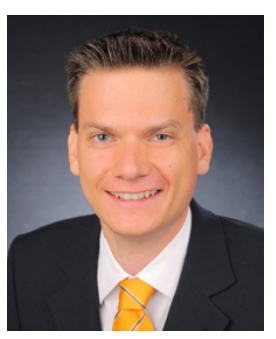

\section{Sebastian Bauer}

University of Wisconsin - Madison, Madison, WI, USA

Karlsruher Institut für Technologie, Institut für Industrielle Informationstechnik, Karlsruhe, Germany sebastian.bauer@kit.edu

Sebastian Bauer hat am Karlsruher Institut für Technologie (KIT) Elektro- und Informationstechnik studiert. Von 2013 bis 2018 arbeitete er als wissenschaftlicher Mitarbeiter am Institut für Industrielle Informationstechnik (IIIT) des KIT. Seine dort angefertigte Dissertation wurde mit dem Messtechnik-Preis 2018 des Arbeitskreises der Hochschullehrer für Messtechnik e. V. ausgezeichnet. Seit Juni 2018 ist er Postdoc in der Computational Optics Group an der University of Wisconsin - Madison, USA.

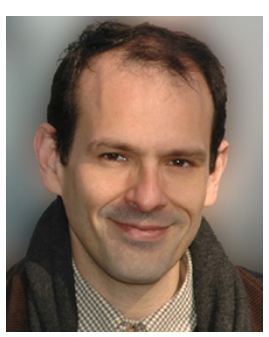

\section{Fernando Puente León}

Karlsruher Institut für Technologie, Institut für Industrielle Informationstechnik, Karlsruhe, Germany fernando.puente-leon@kit.edu

Fernando Puente León leitet das Institut für Industrielle Informationstechnik (IIIT) am Karlsruher Institut für Technologie (KIT). Hauptarbeitsgebiete: Mess- und Automatisierungstechnik, Signalverarbeitung, automatische Sichtprüfung und Bildverarbeitung, Mustererkennung und Informationsfusion. 